\title{
Coup de jeune sur le Grand comité « Notre terreur ", du collectif D'Ores et déjà, mise en scène de Sylvain Creuzevault
}

\section{Serge Aberdam}

\section{(2) OpenEdition \\ 1 Journals \\ Édition électronique \\ URL : https://journals.openedition.org/ahrf/12056 \\ DOI : 10.4000/ahrf.12056 \\ ISSN : 1952-403X \\ Éditeur : \\ Armand Colin, Société des études robespierristes}

\section{Édition imprimée}

Date de publication : 1 juin 2011

Pagination : 243-246

ISSN : 0003-4436

\section{Référence électronique}

Serge Aberdam, «Coup de jeune sur le Grand comité « Notre terreur », du collectif D'Ores et déjà, mise en scène de Sylvain Creuzevault », Annales historiques de la Révolution française [En ligne], 364 | avriljuin 2011, mis en ligne le 01 juin 2014, consulté le 22 avril 2022. URL : http://journals.openedition.org/ ahrf/12056 ; DOI : https://doi.org/10.4000/ahrf.12056 


\section{COUP DE JEUNE SUR LE GRAND COMITÉ "NOTRE TERREUR », DU COLLECTIF D'ORES ET DÉJÀ, MISE EN SC ÈNE DE SYLVAIN CREUZEVAULT}

On ne crée pas tous les jours une pièce de théâtre sur la Révolution française. Après le « Marat-Sade » de Peter Weiss, publié en 1963, traduit par Jean Baudrillard en 1965 et représenté à Paris en 1966-1967¹', les créations les plus marquantes en ce genre depuis quarante ans ont été, en 1970 à Milan puis à Paris en 1972, celle du « 1789 » du Théâtre du Soleil puis en 1972 celle de son « $1793 »$. Ces spectacles ont fait date dans l'ambiance des années 70 , touchant des publics d'une ampleur inusitée. D'autres y ont moins bien réussi, comme «L'affaire de Nancy » de Michel Caffier créée en 1990 au CDN de Lorraine, au Théâtre de la Manufacture et une série bien plus ample d'autres pièces liées au Bicentenaire, données aussi bien par des professionnels que par des amateurs dans tout le pays. Depuis, plus grand chose si l'on met à part, en 1979 et 1988, au Palais des congrès, le « Danton et Robespierre » d'Alain Decaux, Stellio Lorenzi, Georges Soria et Robert Hossein, lourde machine d'un tout autre acabit.

On observe même, ce qui n'est pas si courant, des remords exprimés par l'une des principales créatrices des années 70, Ariane Mnouchkine, interrogée en 2005 par Fabienne Pascaud ${ }^{2}$ :

« J'ai noté que vous n'évoquiez que rarement certains des spectacles, pourtant les plus célèbres du Théâtre du soleil : 1789, 1793, l'Âge d'Or. Comme si vous les aviez oubliés.

A.M. : Parce que c'est trop loin. Et puis il y a un moment où il faut quand même bien que j'ose m'avouer - surtout pour « 1789 » - que c'était simpliste, que je n'aime plus ce style de jeu de bateleur, que je n'accepte-

(1) L'adaptation cinéma du « Marat-Sade », par Peter Brook, est à rapprocher du film tiré $\mathrm{du} \ll 1789 »$.

(2) Ariane Mnouchkine, L'Art au présent, Entretiens avec Fabienne Pascaud, Paris, Plon, 2005 , p. 129. 
rai plus aujourd'hui ces imperfections dans l'interprétation. [...] [le film “1789":] Moi, je ne peux plus le regarder [... " "1793" était moins spectaculaire et donnait à réfléchir sur la tentative de prise en main des affaires publiques par le peuple. On y montrait la vie ordinaire d'une section de sans-culottes de Paris, aux Halles, entre la prise des Tuileries et la destitution du roi, le 10 août 1792, et le début de la Terreur, en septembre 1793. On y voyait comment, pendant plus d'un an, la souveraineté populaire s'était concrètement exercée, via ces sections, véritable avant-garde révolutionnaire et laboratoire d'une démocratie directe [...]»

En regard du succès quasi universel qui fut celui du « 1789 », l'évolution d'Ariane Mnouchkine est frappante même si son cas n'est guère isolé et si on peut par ailleurs comprendre les racines du plus grand intérêt qu'elle manifeste, trente ans plus tard, pour le «1793». Reste que la rupture est marquante et c'est pourquoi il n'est pas anodin d'enregistrer le phénomène que représente aujourd'hui Notre Terreur par le collectif D'Ores et déjà, mis en scène par Sylvain Creuzevault.

Créé en septembre 2009 au Théâtre de la Colline, Notre Terreur a rencontré initialement un réel succès devant le public parisien, confirmé lors de sa reprise un an plus tard et pendant une tournée nationale jusqu'à la fin d'avril 2011. C'est un premier mérite pour un spectacle qui met crânement en avant la question de la Terreur, prise au sens chronologiquement le plus court des quatre mois, entre avril et juillet 1794, qui vont de l'exécution d'Hérault de Séchelles et de Danton et celle de Robespierre. Le comité de Salut public, ramené à neuf membres et occasionnellement rejoint par le fantôme de Danton, débat sous nos yeux de ce que doit être exactement la dictature du même nom et de la politique du gouvernement révolutionnaire dont la Convention l'a chargé et le tiendra pour responsable. Théâtralement, la continuité est réelle avec le «1793» du Soleil, non seulement parce que « la cité révolutionnaire est de ce monde », mais parce que cette nouvelle création collective trace elle aussi un portrait collectif, non plus celui d'une section parisienne mais celui d'un comité qui exerce collectivement le pouvoir.

Le collectif D'Ores et déjà, dont les racines sont indiscutablement libertaires, relève ainsi le défi de traiter des conditions d'exercice de l'autorité, autorité dont les responsabilités sont écrasantes... au point de l'écraser. Incarner ce pouvoir ne facilitait pas l'identification des acteurs avec leurs personnages, même si les uns et les autres ont plus ou moins le même âge, mais ils réussissent à nous faire entrer dans le huis clos des « neuf », portant sur la scène une idée qui était déjà celle de Robert 
Palmer, avec Twelve Who Ruled, ou de Pierre Michon avec son roman Les Onze. Les membres du comité prennent vie, leurs préoccupations et leurs interrelations tumultueuses prennent sens pour le public. Ou bien plutôt cet aspect gagne-il finalement une importance décisive dans la pièce car, d'après la dense présentation de Sylvain Creuzevault qui est distribuée aux spectateurs, son point de départ politique était assez différent.

Il s'agissait plutôt de reconstituer «une question qui regarde Robespierre : est-ce une tyrannie de l'opinion que de se dresser seul contre tous? Soutenir sa conscience, est-ce mettre en défaut l'idée de volonté générale? Ou au contraire peut-elle être pensée, cette solitude d'agir dans laquelle il plonge un instant, comme liberté individuelle politique, un JE en tant que devenir du bien commun? » L'optique de départ prêtait donc à Robespierre une pensée quasi vierge de toute logique étatique, en face de laquelle «L'État français thermidorien se devait de créer une figure qui deviendrait LA violence révolutionnaire inaugurale, LA terreur, LE terroriste. Il devait se couvrir des forfaits que l'histoire lui attribuerait. Il fit mieux, il fit l'histoire. Nous désirons par le théâtre dénoncer cette imposture, lui rebrousser le poil; ce n'est pas un jugement aveugle mais un désir de distinction ». Ce désir de distinction n'est pas en cause : son expression est la condition même d'un débat public digne de ce nom, même si la rêverie historique a tendance à remplacer l'analyse (« Le neuf Thermidor, ON empêchera Robespierre de revenir au NOUS ») et à opposer un personnage mythifié à un autre :

«Aujourd'hui, c'est très commode. La chute du bloc soviétique a opéré comme un choc symbolique, et les processus révolutionnaires en général, les luttes armées en particulier, et Robespierre en tant qu'individu inaugural, sont devenus des cibles consensuelles exquises, ON peut y tirer à l'aveugle ».

Le lecteur pourrait surtout croire qu'il s'agit à nouveau de réparer au théâtre une injustice historique : « Notre Terreur décrie et révoque ironiquement cette transmission de la figure de Robespierre. Ironiquement, car justement cette figure n'a pas à être transmise dans cette solitude excessive $\gg$.

Le travail collectif me semble pourtant avoir tiré le meilleur parti de l'option idéologique libertaire d'une "circulation insurrectionnelle instable contre laquelle aucune loi [...] ne peut rien ». Cette liberté de la troupe, travaillant intensément les ouvrages disponibles, a permis de donner toute leur importance aux tâches que les neuf personnages considéraient comme les leurs et à ce qu'ils pouvaient bien en penser. Le résultat 
me semble frappé d'un indiscutable talent. En d'autres termes, la création collective a entraîné un résultat peut-être assez différent du projet initial, centré sur le seul incorruptible, recentrage admis par nombre de participants, et on me permettra de penser que c'est à la fois original et heureux.

La confrontation entre les "neuf » qui s'essaient à résoudre une série de questions concrètes de l'époque, sonne presque aussi juste qu'en son temps le «1793» du Soleil" . Certaines scènes de " Notre Terreur» font invinciblement penser à d'autres huis clos, ceux de jeunes directions politiques en pleines crises révolutionnaires, traversées de sympathies et de déchirements. La pièce est donc une réflexion sur les collectifs agissants et sur le pouvoir partagé, elle laisse ouverte une multiplicité d'approches de la Terreur et reste finalement formidablement tonique. Elle n'est évidemment pas exempte de maladresses : on répand sur scène trop de peinture, blanche ou rouge, et on sent encore que le traitement de Robespierre n'en finit pas d'avoir été discuté et rediscuté mais le résultat, c'est de sortir le sujet de la naphtaline du prêt à penser. Je souhaite à nos lecteurs de retrouver la fraîcheur et la force de jeu que le spectacle avait fin septembre, lorsque je l'ai vu, mais je crois tout aussi important de réfléchir à ce que peut signifier cette appropriation par un groupe de jeunes de la situation du Grand comité.

Serge ABERDAM

(3) Denis Bablet et Marie-Louise BABlet, Le Théâtre du Soleil ou la quête du bonheur, diapolivre, Paris, Editions du CNRS, 1979, pp. 58-73; disponible sur le site du Théâtre du Soleil. 